\title{
Aprendizaje basado en el planteamiento de casos clínicos en Contactología
}

\section{Learning based on the presentation of clinical report in Contactology}

MARÍA JOSÉ BAUTISTA LLAMAS

ORCID: https://orcid.org/0000-0001-8317-5014

Universidad de Sevilla

Dpto. Física de la Materia Condensada

mbautista@us.es

Fecha de recepción: 03-06-2019.

Fecha de aceptación:14-06-2019.

DOI: http://dx.doi.org/10.12795/9788447221912.011

Pp.: 267-287 


\section{Resumen}

Este artículo describe la puesta en práctica de un ciclo de mejora en el aula aplicado en la asignatura Contactología II, que se imparte de manera conjunta en el Grado en Óptica y Optometría y en el Doble Grado en Farmacia y en Óptica y Optometría de la Facultad de Farmacia de la Universidad de Sevilla. Este ciclo de mejora se centra en la docencia correspondiente a la selección de pacientes y la lente de contacto adecuada para cada uno de ellos, así como las complicaciones derivadas de las mismas.

El modelo metodológico se basa en el planteamiento de una pregunta sobre el tema que se pretende aprender por parte del profesor, y que esa pregunta obtenga respuesta a través de la resolución de un problema o caso práctico planteado y desarrollado por los alumnos, y expuesto en clase para discutir y extraer conclusiones sobre el mismo.

Palabras Clave: Contactología, Óptica y Optometría, docencia universitaria, experimentación docente universitaria, caso clínico.

\section{Abstract}

This paper is based in a Improvement Cycle in Classroom (ICIC) applied in the subject Contactology II, which is taught both in the Degree in Optics and Optometry and the Double Degree in Pharmacy and Optics and Optometry of the Faculty of Pharmacy at the University of Seville. The patient selection and contact lens suitable for each of them, as well as the complications for use of contact lenses is the objective of this ICIC.

The methodological model is based on the approach of a question on the subject that you want to work, and this question will get an answer through the resolution of a problem or case report proposed and developed by the students. This case report is exposed in class for discuss and draw conclusions.

Keywords: Contactology, Optics and Optometry, university teaching, university teaching experimentation, clinical report. 


\section{Contexto}

En este artículo se describe la aplicación de un ciclo de mejora en el aula (CIMA) realizado en la asignatura de Contactología II, que se imparte en tercero del Grado de Òptica y Optometría, y en quinto del Doble Grado de Farmacia y Óptica y Optometría de la Facultad de Farmacia en la Universidad de Sevilla, en donde hay 60 y 20 alumnos matriculados respectivamente durante el curso 2018/2019.

Estos alumnos ya han cursado Contactología I, por lo que tienen conocimientos de lentes de contacto y sobre la valoración de pacientes previa a la adaptación. La finalidad de esta asignatura es profundizar en la selección de pacientes y la metodología de adaptación de diferentes diseños en función de las caracteristicas de cada persona.

En este momento, en la asignatura, ya se han explicado todos los materiales y diseños de lentes que existen de forma teórica. El tema en el que se va a aplicar el ciclo de mejora es el de selección de pacientes y complicaciones post adaptación de las mismas.

\section{Diseño del CIMA}

\section{Modelo metodológico}

La metodología aplicada hasta el momento en la asignatura de Contactología II consiste en que la profesora imparte una clase magistral y propone problemas a los alumnos que son resueltos en clase. La intención con el CIMA es cambiar este modelo, de forma que sean los alumnos los que planteen sus casos, así como su solución, y posibles complicaciones en la adapción de lentes de contacto, a la vez de que sean capaces de discutirlo con sus compañeros. 
Por tanto, en el modelo metodológico posible, me baso en una pregunta sobre el tema que se pretende que aprendan, y al que los alumnos deben dar respuesta a través de la resolución de un problema o caso práctico. Para ello trabajarán en grupos mientras resuelvo de forma individual sus preguntas para ayudales a avanzar.

En la figura 1 podemos observar el esquema de este modelo, en el que el grueso de la clase lo ocuparía el trabajo de los alumnos. De las soluciones y conclusiones aportadas debería poder extraerse una nueva pregunta que nos permita avanzar en conocimientos, es decir, un modelo basado en el constructivismo, en el que partiendo de lo conocimientos previos de los alumnos lleguemos a un aprendizaje superior (Coll et al., 1993; Cubero, 2005).

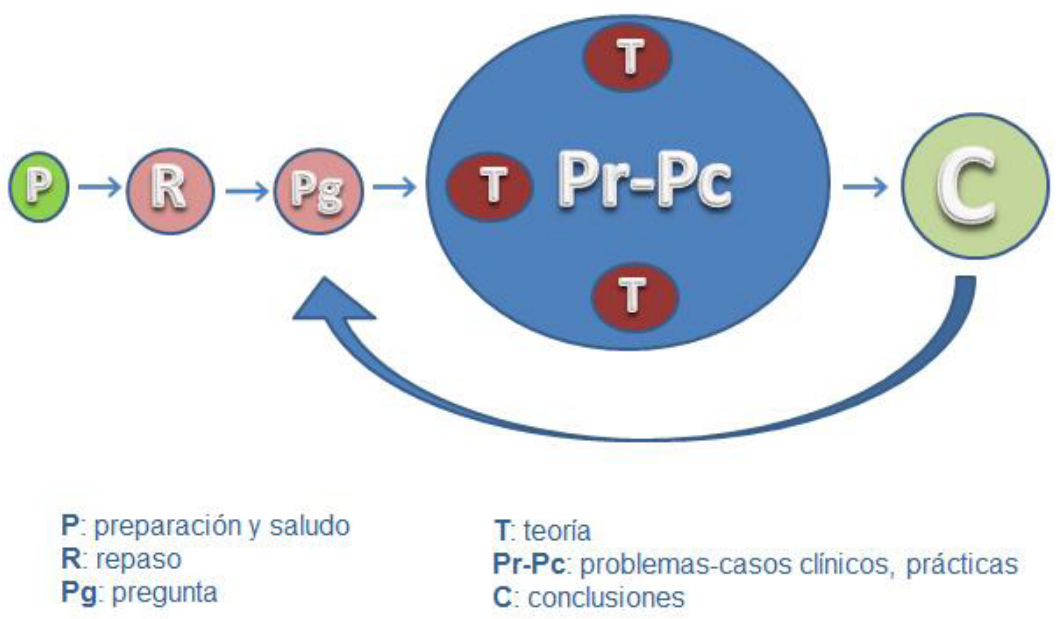

Figura 1. Modelo metodológico posible

Respecto a mi metodología actual, pretendo no tener que ir planteando yo las preguntas, si no que sean los propios alumnos quienes la planteen según vayan profundizando en la resolución del caso que tienen delante, lo cual se asemejaría a la puesta en práctica en la vida real de esta asignatura (Finkel, 2008). 
Otro de los retos es que los alumnos sean resolutivos y críticos, discutan la solución con sus compañeros, y la defiendan según sus conocimientos; que no asuman como propias las directrices marcadas por un profesor en una clase magistral, si no que entiendan que pueden no opinar igual, o no trabajar de la misma manera.

\section{Mapa de contenidos}

Como ya he comentado, el CIMA tiene como objetivo que los alumnos aprendan a seleccionar de forma adecuada los posibles usuarios de lentes de contacto, y la lente adecuada para cada uno de ellos. Ya tienen asumido el proceso de adaptación, con lo cual el siguiente paso sería determinar un plan de seguimiento, y poder identificar las complicaciones que puedan surgir. Para ello la pregunta que se les plantea es: "¿Todos podemos usar lentes de contacto?", y de esta pregunta parten flechas hasta todas las pruebas que determinan si se pueden adaptar lentes, y que están unidas por un círculo, puesto que los resultado obtenidos en todas ellas darán lugar al tipo de lente de contacto adaptada (García-Díaz et al., 2017). El siguiente paso sería la adaptación y seguimiento de las mismas, que aparecen unidas a las pruebas que deben realizarse de nuevo en cada visita de seguimiento (figura 2).

Jornadas de Formación e Innovación Docente del Profesorado | № 2 (2019) Esta obra se distribuye con la licencia Creative Commons Reconocimiento-NoComercial-SinObraDerivada 4.0 Internacional (CC BY-NC-ND 4.0.) 


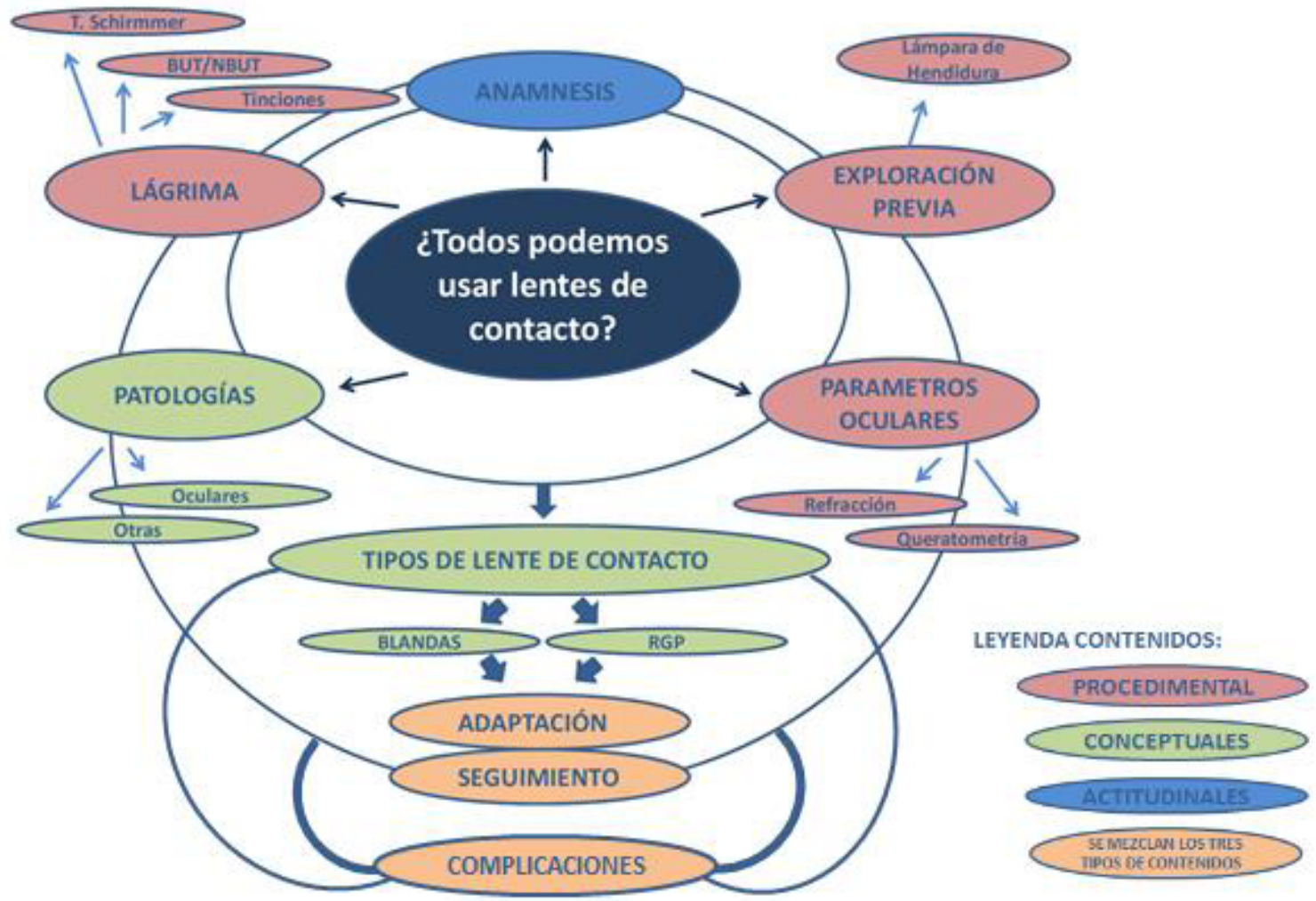

Figura 2. Mapa de contenidos

Jornadas de Formación e Innovación Docente del Profesorado | № 2 (2019)

cC (i) Esta obra se distribuye con la licencia Creative Commons 
Por último, nos encontramos con las complicacionesque puedan surgir y que dependerán del tipo de lente de contacto y, a su vez, del seguimiento que se realice de la adaptación, por tanto, de las pruebas exploratorias a las que se somete al paciente.

\section{Secuencia de actividades}

La aplicación del CIMA se ha llevado a cabo en cuatro sesiones de una hora y media, siendo necesaria la planificación de todas las actividades que se van a llevar a cabo en cada una. La secuencia queda reflejada en las tablas 1 y 2.

Tabla1. Secuencia de actividades en la aplicación del CIMA (sesiones 1 y 2 )

\begin{tabular}{|l|l|l|l|}
\hline \multicolumn{5}{|c|}{ PRIMERA SESIÓN } \\
\hline $\begin{array}{c}\text { ACTIVIDAD/ } \\
\text { TIEMPO }\end{array}$ & \multicolumn{1}{|c|}{$\begin{array}{c}\text { DESARROLLO DE LA } \\
\text { ACTIVIDAD }\end{array}$} & FINALIDAD & CONTENIDOS \\
\hline $\begin{array}{l}\text { Presentar el } \\
\text { ejercicio (10 } \\
\text { min) }\end{array}$ & $\begin{array}{l}\text { Explicar a los alumnos } \\
\text { como se van a desarrollar } \\
\text { las próximas clases. Tra- } \\
\text { bajando en grupo y par- } \\
\text { tiendo de parámetros } \\
\text { oculares plantear dos su- } \\
\text { puestos pacientes, con } \\
\text { diferentes caracteristi- } \\
\text { cas (diferente anamnesis, } \\
\text { motivación y plan de uso) } \\
\text { que les conduzca a re- } \\
\text { comendar y calcular una } \\
\text { lente de contacto. }\end{array}$ & $\begin{array}{l}\text { Selección de } \\
\text { trabajo. } \\
\text { pacientes y } \\
\text { elección de } \\
\text { la lente de } \\
\text { contacto. }\end{array}$ \\
\hline $\begin{array}{l}\text { Resolver du- } \\
\text { das (5 min) }\end{array}$ & $\begin{array}{l}\text { Responder a las pregun- } \\
\text { tas de los alumnos. }\end{array}$ & \\
\hline
\end{tabular}

Jornadas de Formación e Innovación Docente del Profesorado | № 2 (2019) Esta obra se distribuye con la licencia Creative Commons Reconocimiento-NoComercial-SinObraDerivada Internacional (CC BY-NC-ND 4.0.) 


\begin{tabular}{|c|c|c|c|}
\hline $\begin{array}{l}\text { Responder } \\
\text { cuestionario } \\
\text { (15 min) }\end{array}$ & $\begin{array}{l}\text { Cada alumno responde } \\
\text { al cuestionario de forma } \\
\text { corta y clara. }\end{array}$ & $\begin{array}{l}\text { Determinar } \\
\text { el nivel de } \\
\text { conocimien- } \\
\text { tos de los } \\
\text { alumnos. }\end{array}$ & \\
\hline $\begin{array}{l}\text { Crear gru- } \\
\text { pos de } \\
\text { trabajo } \\
\text { ( } 5 \text { min) }\end{array}$ & $\begin{array}{l}\text { Los alumnos hacen gru- } \\
\text { pos de trabajo. }\end{array}$ & $\begin{array}{l}\text { Fomen- } \\
\text { tar el inter- } \\
\text { cambio de } \\
\text { opiniones. }\end{array}$ & \\
\hline $\begin{array}{l}\text { Trabajar en } \\
\text { grupo para } \\
\text { plantear las } \\
\text { posibles so- } \\
\text { luciones del } \\
\text { ejercicio } \\
\text { (40 min) }\end{array}$ & $\begin{array}{l}\text { Con los parámetros ocu- } \\
\text { lares (queratometría, } \\
\text { refracción, diámetro cor- } \\
\text { neal, diámetro pupilar) } \\
\text { deberán trabajar en dos } \\
\text { supuestos pacientes a los } \\
\text { que deben añadir carac- } \\
\text { terísticas y que les lleve a } \\
\text { dos indicaciones de len- } \\
\text { tes de contacto. }\end{array}$ & $\begin{array}{l}\text { Seleccio- } \\
\text { nar la lente } \\
\text { de contacto } \\
\text { adecuada } \\
\text { en función } \\
\text { de las ca- } \\
\text { racterísti- } \\
\text { cas de cada } \\
\text { paciente. }\end{array}$ & \\
\hline $\begin{array}{l}\text { Dar instruc- } \\
\text { ciones para } \\
\text { la siguiente } \\
\text { clase } \\
\text { (5 min) }\end{array}$ & $\begin{array}{l}\text { El profesor explica cómo } \\
\text { se trabajará el próximo } \\
\text { día de clase. }\end{array}$ & $\begin{array}{l}\text { Cerrar sesión } \\
\text { y preparar la } \\
\text { siguiente. }\end{array}$ & \\
\hline \multicolumn{4}{|c|}{ SEGUNDA SESIÓN } \\
\hline $\begin{array}{l}\text { Repasar } \\
\text { y seguir } \\
\text { trabajando } \\
\text { (5 min) }\end{array}$ & $\begin{array}{l}\text { Breve explicación sobre la } \\
\text { clase anterior. }\end{array}$ & $\begin{array}{l}\text { Poner en si- } \\
\text { tuación a } \\
\text { los alumnos } \\
\text { para la ac- } \\
\text { tividad de } \\
\text { hoy. }\end{array}$ & \\
\hline $\begin{array}{l}\text { Taller para } \\
\text { reconocer } \\
\text { lentes de } \\
\text { contacto } \\
\text { (40 min) }\end{array}$ & $\begin{array}{l}\text { Se les entregan lentes de } \\
\text { contacto de diferentes ca- } \\
\text { racterísticas (blandas de } \\
\text { diferentes reemplazo y rí- } \\
\text { gidas gas permeable), } \\
\text { para que las manipulen. }\end{array}$ & $\begin{array}{l}\text { Manipulando } \\
\text { las lentes de } \\
\text { contacto to- } \\
\text { marán con- } \\
\text { ciencia de } \\
\text { las carac- } \\
\text { terísticas y } \\
\text { aplicarlo a la } \\
\text { práctica. }\end{array}$ & $\begin{array}{l}\text { Caracterís- } \\
\text { ticas de las } \\
\text { lentes de } \\
\text { contacto. }\end{array}$ \\
\hline
\end{tabular}

Jornadas de Formación e Innovación Docente del Profesorado | № 2 (2019) Esta obra se distribuye con la licencia Creative Commons 


\begin{tabular}{|l|l|l|l|}
\hline $\begin{array}{l}\text { Revisar las } \\
\text { soluciones } \\
\text { 40 min) }\end{array}$ & $\begin{array}{l}\text { Debatir las soluciones } \\
\text { aportadas tras asumir los } \\
\text { conceptos de sus caracte- } \\
\text { rísticas en el taller. }\end{array}$ & $\begin{array}{l}\text { Replantear } \\
\text { los conoci- } \\
\text { mientos que } \\
\text { dieron por } \\
\text { asumidos. }\end{array}$ & \\
\hline $\begin{array}{l}\text { Dar instruc- } \\
\text { ciones para } \\
\text { la siguiente } \\
\text { clase } \\
\text { (5 min) }\end{array}$ & $\begin{array}{l}\text { El profesor explica cómo } \\
\text { se desarrollará la si- } \\
\text { guiente clase en la que se } \\
\text { expondrán los resultados. }\end{array}$ & $\begin{array}{l}\text { Conocer el } \\
\text { desarro- } \\
\text { llo de la si- } \\
\text { guiente } \\
\text { clase. }\end{array}$ & \\
\hline
\end{tabular}

Tabla 2. Secuencia de actividades en la aplicación del CIMA (sesiones 3 y 4 )

\begin{tabular}{|c|c|c|c|}
\hline \multicolumn{4}{|c|}{ TERCERA SESIÓN } \\
\hline $\begin{array}{l}\text { ACTIVIDAD / } \\
\text { TIEMPO }\end{array}$ & $\begin{array}{l}\text { DESARROLLO DE LA } \\
\text { ACTIVIDAD }\end{array}$ & FINALIDAD & CONTENIDOS \\
\hline $\begin{array}{l}\text { Repasar la } \\
\text { clase anterior } \\
\text { (5 min) }\end{array}$ & $\begin{array}{l}\text { Breve explicación sobre } \\
\text { la clase anterior. }\end{array}$ & $\begin{array}{l}\text { Poner en si- } \\
\text { tuación a los } \\
\text { alumnos. }\end{array}$ & \multirow{2}{*}{$\begin{array}{l}\text { Selección } \\
\text { de pacien- } \\
\text { tes y elección } \\
\text { de la lente de } \\
\text { contacto. }\end{array}$} \\
\hline $\begin{array}{l}\text { Exponer los re- } \\
\text { sultados del } \\
\text { trabajo en } \\
\text { grupo ( } 45 \mathrm{~min})\end{array}$ & $\begin{array}{l}\text { Se exponen los supues- } \\
\text { tos que han desarro- } \\
\text { llado y se debaten con } \\
\text { el resto de alumnos. }\end{array}$ & $\begin{array}{l}\text { Selección de la } \\
\text { lente de con- } \\
\text { tacto según el } \\
\text { paciente. }\end{array}$ & \\
\hline $\begin{array}{l}\text { Explicar si- } \\
\text { guiente } \\
\text { actividad } \\
\text { (5 min) }\end{array}$ & $\begin{array}{l}\text { Trabajar en grupo para } \\
\text { establecer un plan de } \\
\text { seguimiento según } \\
\text { la lente de contacto } \\
\text { adaptada. }\end{array}$ & & \multirow[t]{2}{*}{$\begin{array}{l}\text { Establecer cri- } \\
\text { terios de se- } \\
\text { guimiento tras } \\
\text { adaptación } \\
\text { de lentes de } \\
\text { contacto. }\end{array}$} \\
\hline $\begin{array}{l}\text { Trabajar en } \\
\text { grupo para es- } \\
\text { tablecer el se- } \\
\text { guimiento de } \\
\text { un paciente } \\
\text { con lentes de } \\
\text { contacto ( } 30 \\
\text { min) } \\
\end{array}$ & $\begin{array}{l}\text { Por grupos trabajarán } \\
\text { el seguimiento del pa- } \\
\text { ciente tras la adap- } \\
\text { tación de lentes de } \\
\text { contacto. }\end{array}$ & \begin{tabular}{|l} 
Entender que \\
cada lente de \\
contacto puede \\
tener un efecto \\
diferente en el \\
ojo y por tanto \\
un seguimiento \\
diferente. \\
\end{tabular} & \\
\hline $\begin{array}{l}\text { Dar instruccio- } \\
\text { nes para la si- } \\
\text { guiente clase } \\
\text { (5 min) }\end{array}$ & $\begin{array}{l}\text { En la siguiente clase } \\
\text { se expondrán y debati- } \\
\text { rán los resultados de la } \\
\text { clase de hoy. }\end{array}$ & $\begin{array}{l}\text { Que los alum- } \\
\text { nos preparen las } \\
\text { exposiciones. }\end{array}$ & \\
\hline
\end{tabular}

Jornadas de Formación e Innovación Docente del Profesorado | № 2 (2019) Esta obra se distribuye con la licencia Creative Commons Reconocimiento-NoComercial-SinObraDerivada Internacional (CC BY-NC-ND 4.0.) 


\section{CUARTA SESIÓN}

\begin{tabular}{|c|c|c|c|}
\hline $\begin{array}{l}\text { Repasar la } \\
\text { clase anterior } \\
(5 \mathrm{~min})\end{array}$ & $\begin{array}{l}\text { Breve explicación sobre } \\
\text { la clase anterior. }\end{array}$ & $\begin{array}{l}\text { Poner en si- } \\
\text { tuación a los } \\
\text { alumnos. } \\
\end{array}$ & \multirow{3}{*}{$\begin{array}{l}\text { Establecer cri- } \\
\text { terios de se- } \\
\text { guimiento tras } \\
\text { adaptación } \\
\text { de lentes de } \\
\text { contacto. }\end{array}$} \\
\hline $\begin{array}{l}\text { Exponer los re- } \\
\text { sultados del } \\
\text { trabajo en } \\
\text { grupo. ( } 40 \mathrm{~min})\end{array}$ & $\begin{array}{l}\text { El representante de } \\
\text { cada grupo expone los } \\
\text { supuestos que han de- } \\
\text { sarrollado en cuanto al } \\
\text { seguimiento a realizar } \\
\text { tras la adaptación de } \\
\text { lentes de contacto y se } \\
\text { debaten con el resto de } \\
\text { alumnos. }\end{array}$ & $\begin{array}{l}\text { Conocer los di- } \\
\text { ferentes criterios } \\
\text { de seguimiento } \\
\text { tras la adapta- } \\
\text { ción de lentes } \\
\text { de contacto. }\end{array}$ & \\
\hline $\begin{array}{l}\text { Extraer } \\
\text { conclusiones } \\
(20 \text { min })\end{array}$ & $\begin{array}{l}\text { La profesora resume } \\
\text { con la ayuda de los } \\
\text { alumnos las últimas } \\
\text { clases. }\end{array}$ & $\begin{array}{l}\text { Desarro- } \\
\text { llar la capaci- } \\
\text { dad de extraer } \\
\text { conclusiones. }\end{array}$ & \\
\hline $\begin{array}{l}\text { Explicar y rea- } \\
\text { lizar cuestio- } \\
\text { nario final } \\
(20 \mathrm{~min})\end{array}$ & $\begin{array}{l}\text { Cada alumno responde } \\
\text { al cuestionario final de } \\
\text { forma corta y clara. }\end{array}$ & $\begin{array}{l}\text { Determinar el } \\
\text { nivel de conoci- } \\
\text { mientos de los } \\
\text { alumnos des- } \\
\text { pués de la apli- } \\
\text { cación del ciclo }\end{array}$ & $\begin{array}{l}\text { Selección } \\
\text { de pacien- } \\
\text { tes y elección } \\
\text { de la lente de } \\
\text { contacto. }\end{array}$ \\
\hline $\begin{array}{l}\text { Cerrar la } \\
\text { actividad } \\
(5 \text { min })\end{array}$ & $\begin{array}{l}\text { La profesora agradece } \\
\text { la participación de los } \\
\text { alumnos y da por termi- } \\
\text { nada la actividad. }\end{array}$ & & \\
\hline
\end{tabular}

\section{Aplicación del CIMA}

Comienza el CIMA con la explicación del proceso que vamos a desarrollar durante las siguientes clases, un trabajo en grupo en el que yo les doy a los alumnos parte de los datos de un examen realizado a un paciente, y deberán completar los que les faltan orientándolo a un tipo de adaptación concreta. Después los expondrán y debatirán con el resto de la clase para aclarar conceptos.

Para motivarles les explico que entre los casos expuestos se elegirán los más trabajados y completos y obtendrán 
puntuación extra en el examen parcial. Tomo esta medida para motivar a un sector de la clase que es poco participativa y que presenta poco interés en general.

Antes de que se pongan a trabajar les entrego un cuestionario, al que pueden responder de forma voluntaria, con su nombre o un pseudónimo, y que aclaro, nos va a ayudar a conocer si sus conceptos después de trabajarlos en grupo están más claros o no.

En la segunda sesión doy a cada grupo una muestra de lentes de diferentes materiales (hidrogel, hidrogel-silicona, hidrogel-silicona -uso diario- y Rígida Gas Permeable), les pido que las manipulen y que valoren diferencias $y$, por medio de preguntas y con las dudas que les surgen, tengan una idea clara de cómo funciona cada material. Hasta ahora conocían las propiedades teóricas de cada tipo, pero quiero que sean conscientes de las mismas in vivo, y que repasen la indicación de sus casos clínicos. Este taller les resulta muy interesante, empiezan a tomar conciencia de la relación entre sus conocimientos teóricos y la práctica.

Entre la segunda y tercera sesión se exponen todos los trabajos. En general son buenos, no profundizan mucho en sus indicaciones, pero eso me da pie a establecer debate y que todos puedan opinar.

A raíz de las dudas planteadas con los materiales, en la tercera sesión me llevé a clase tarifas de un par de casas comerciales de lentes de contacto donde se describen los productos de cada una de ellas, diferentes materiales, uso y parámetros. En uno de los casos plantean la adaptación de una lente diaria cuyos parámetros no se fabrican, entonces les paso las tarifas y los emplazo a buscar esa lente que necesitan, y que no encuentran, tomando conciencia de las limitaciones que la práctica real pone a la teoría. 
En todas las actividades planificadas hemos superado el tiempo previsto, por lo que para la cuarta sesión trabajan en casa las complicaciones surgidas por el uso de lentes de contacto, siendo esta sesión menos productiva que las anteriores. Las exposiciones son más escuetas y el interés de los alumnos por el tema es menor, aun así, necesitamos una sesión más de las previstas para terminar con las exposiciones, extraer conclusiones y volver a contestar al cuestionario. De todas formas, su participación me ha sido de gran ayuda como hilo conductor, aunque creo que en el diseño de actividades me equivoqué al repetir el trabajo en grupo y la exposición oral de los resultados de forma tan seguida.

Para evaluar la efectividad del CIMA, y siguiendo las directrices recogidas en Rivero y Porlán (2017), elaboré un cuestionario para pasarlo a los estudiantes antes y después del ciclo. Estas son las preguntas formuladas a los estudiantes:

1. De las personas que te rodean, ¿a quién no le pondrías lentes de contacto y por qué?

2. Mi vecino me ha dicho que las lentes de contacto le han provocado una infección: ¿eso es posible?, ¿qué factores habrán influido?

3. Tu prima no quiere usar lentes de contacto porque piensa que va a perder la visión: ¿crees que es una posibilidad real?, ¿por qué?

4. En un vuelo que realicé hace un mes el pasajero que estaba a mi lado se quitó las lentes de contacto antes de despegar. Según tus conocimientos: ¿se pueden usar lentes de contacto en un avión?, ¿por qué?

5. Cuando era más joven mis padres me decían que no usara lentes de contacto, que solo son recomendables para adultos. ¿Cuál es tu opinión? ¿Se puede usar cualquier lente de contacto a cualquier edad? ¿Por qué? 
Las respuestas son clasificadas en función de los diferentes niveles de aprendizaje identificados y descritos en la figura 3. Estos niveles se han establecido en función de si los alumnos optaban por respuestas simples o iban añadiendo información detallada y justificada a las mismas.

Tabla 3. Clasificación de las respuestas obtenidas en el cuestionario

\begin{tabular}{|c|c|c|c|c|}
\hline PREGUNTA & A & B & C & D \\
\hline $\begin{array}{l}\text { De las personas } \\
\text { que te rodean, ¿a } \\
\text { quién no le pon- } \\
\text { drías lentes de } \\
\text { contacto y por } \\
\text { qué? }\end{array}$ & $\begin{array}{l}\text { Motivación, } \\
\text { mala hi- } \\
\text { giene o gra- } \\
\text { duaciones } \\
\text { fuera de } \\
\text { norma. }\end{array}$ & Ojo seco & $\begin{array}{l}\text { Patologías } \\
\text { que modifi- } \\
\text { quen la fisio- } \\
\text { logía ocular }\end{array}$ & $\begin{array}{l}\text { Hacen re- } \\
\text { ferencia a } \\
\text { la gran va- } \\
\text { riedad de lc } \\
\text { que existen, } \\
\text { hay pocas } \\
\text { exclusiones. }\end{array}$ \\
\hline $\begin{array}{l}\text { Mi vecino me ha } \\
\text { dicho que las len- } \\
\text { tes de contacto } \\
\text { le han provocado } \\
\text { una infección: ¿eso } \\
\text { es posible?, ¿qué } \\
\text { factores habrán } \\
\text { influido? }\end{array}$ & No & $\begin{array}{l}\text { Sí, por } \\
\text { depósi- } \\
\text { tos en las } \\
\text { lc o mala } \\
\text { higiene } \\
\text { personal. }\end{array}$ & $\begin{array}{l}\text { Sí, igual que } \\
\text { B+No cumplir } \\
\text { los reempla- } \\
\text { zos de la lc. }\end{array}$ & $\begin{array}{l}\text { Sí, A+B+ } \\
\text { lc mal } \\
\text { adaptada. }\end{array}$ \\
\hline $\begin{array}{l}\text { Tu prima no quiere } \\
\text { usar lentes de } \\
\text { contacto porque } \\
\text { piensa que va a } \\
\text { perder la visión: } \\
\text { ¿crees que es una } \\
\text { posibilidad real? } \\
\text { ¿Por qué? }\end{array}$ & No & $\begin{array}{l}\text { Sí, pero } \\
\text { es algo } \\
\text { muy raro } \\
\text { y muy } \\
\text { poco } \\
\text { frecuente. }\end{array}$ & $\begin{array}{l}\text { Sí, } \\
\text { infecciones. }\end{array}$ & $\begin{array}{l}\text { Sí, infeccio- } \\
\text { nes (Acan- } \\
\text { thamoeba), } \\
\text { no cumplir } \\
\text { las pautas } \\
\text { de uso yo } \\
\text { reemplazo. }\end{array}$ \\
\hline
\end{tabular}

Jornadas de Formación e Innovación Docente del Profesorado | № 2 (2019) Esta obra se distribuye con la licencia Creative Commons Reconocimiento-NoComercial-SinObraDerivada Internacional (CC BY-NC-ND 4.0.) 


\begin{tabular}{|c|c|c|c|c|}
\hline $\begin{array}{l}\text { En un vuelo que } \\
\text { realicé hace un } \\
\text { mes el pasajero } \\
\text { que estaba a mi } \\
\text { lado se quitó las } \\
\text { lentes de contacto } \\
\text { antes de despegar. } \\
\text { Según tus conoci- } \\
\text { mientos: ¿Se pue- } \\
\text { den usar lentes } \\
\text { de contacto en un } \\
\text { avión? ¿Por qué? }\end{array}$ & $\begin{array}{l}\text { No sabe/no } \\
\text { contesta }\end{array}$ & Sí/No & $\begin{array}{l}\text { Por los cam- } \\
\text { bios de pre- } \\
\text { sión del } \\
\text { avión (no es } \\
\text { la correcta } \\
\text { pero se plan- } \\
\text { tea que } \\
\text { puede afectar } \\
\text { al material } \\
\text { de la lc) }\end{array}$ & $\begin{array}{l}\text { Sí, en fun- } \\
\text { ción de la } \\
\text { duración } \\
\text { del viaje, el } \\
\text { manteni- } \\
\text { miento de } \\
\text { la lc, si se } \\
\text { le seca con } \\
\text { aire acondi- } \\
\text { cionado y/o } \\
\text { se duerme. }\end{array}$ \\
\hline $\begin{array}{l}\text { Cuando era más } \\
\text { joven mis padres } \\
\text { me decían que no } \\
\text { usara lentes de } \\
\text { contacto, que solo } \\
\text { son recomenda- } \\
\text { bles para adultos. } \\
\text { ¿Se puede usar } \\
\text { cualquier lente de } \\
\text { contacto a cual- } \\
\text { quier edad? ¿Por } \\
\text { qué? }\end{array}$ & No & $\begin{array}{l}\text { Sí, si se } \\
\text { implica } \\
\text { en la } \\
\text { adapta- } \\
\text { ción. }\end{array}$ & $\begin{array}{l}\text { Sí, en función } \\
\text { de sus ca- } \\
\text { racterísticas } \\
\text { (parámetros } \\
\text { oculares y } \\
\text { refracción). }\end{array}$ & $\begin{array}{l}\text { Sí, } \mathrm{B}+\mathrm{C}+- \\
\text { control de } \\
\text { miopía, tra- } \\
\text { tamiento de } \\
\text { ambliopía } \\
\text { y/o aniso- } \\
\text { metropías. }\end{array}$ \\
\hline
\end{tabular}

\title{
Resultados del cuestionario inicial y final. Escaleras de aprendizaje
}

\author{
El cuestionario fue respondido por 57 alumnos al inicio
} del ciclo y por 49 al final, de los que 41 coincidieron en ambos, por lo que descarto todos los que no han respondido a los dos para poder establecer una comparación.

Analizando las respuestas, en la pregunta 1, “De las personas que te rodean, ¿a quién no le pondrías lentes de contacto y por qué?" es donde he encontrado el nivel más bajo, tal vez no fueron capaces de identificar que debían excluir usuarios por patologías que lo limitaran, y la mayoría descartaron por no saber manipularlas, sin darse cuenta de que ellos mismos no las manipulan bien, deben aprender. Este punto fue bastante comentado en clase. En la figura 4 podemos observar cómo más de la mitad de los 
alumnos se sitúan en el escalón más bajo en el cuestionario inicial, porcentaje que pasa a ocupar el tercero de cuatro en el cuestionario final.

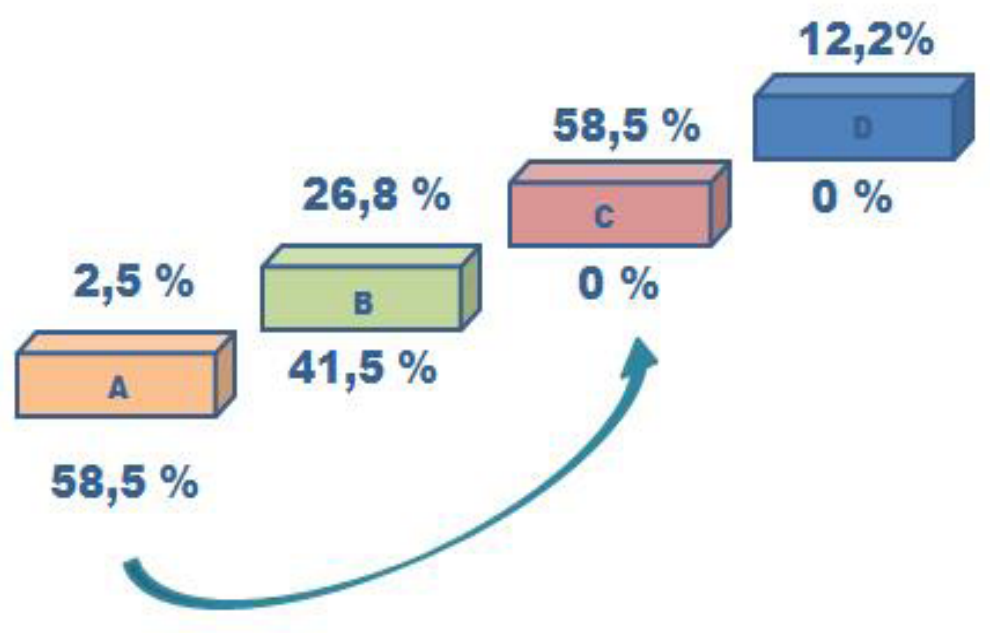

Figura 3. Escalera de aprendizaje para la pregunta 1

Para la pregunta dos, la respuesta a si las lentes de contacto podían provocar una infección la tenían bastante clara, aunque no de qué tipo, o si podía influir algún factor aparte de la limpieza, como era el uso ilimitado de las mismas. El mayor porcentaje de respuestas se encuentra en el segundo escalón y pasan al tercero (figura 5) en el cuestionario final. Esta vez el cambio en los conceptos no es tan evidente, y el porcentaje de alumnos situados en el nivel más bajo no ha cambiado. El tema es muy importante y debería haber quedado bastante más claro, la mayoría de los alumnos deberían haber alcanzado el nivel más alto, por tanto tendré que replantearme la forma de tratar el tema y la formulación de la pregunta. 


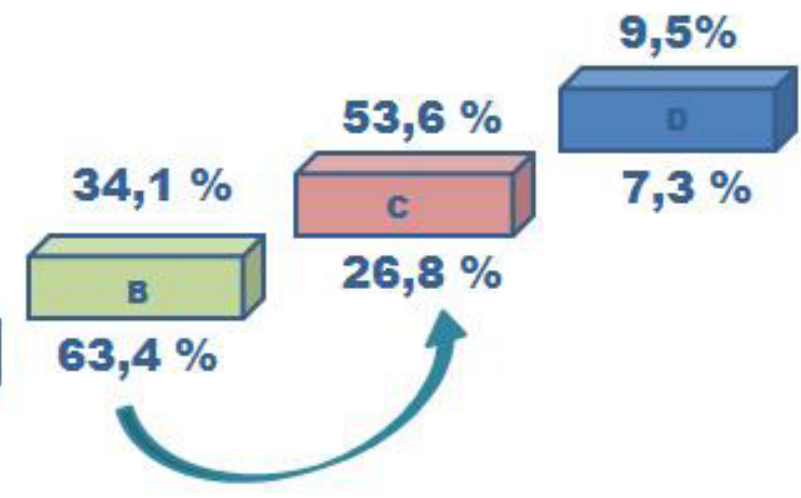

Figura 4. Escalera de aprendizaje para la pregunta 2

En la pregunta 3 se observa un mayor porcentaje en el escalón más bajo y pasa al tercero en el cuestionario final (figura 6). Muestran conocimientos sobre las posibles infecciones que provocan las lentes de contacto, pero no tienen tan claro cuáles cursan con una pérdida transitoria de la agudeza visual o definitiva. El nivel de aprendizaje alcanzado en esta cuestión debería ser mayor, vuelve a poner de manifiesto que no quedan claras las complicaciones derivadas del uso de lentes de contacto.

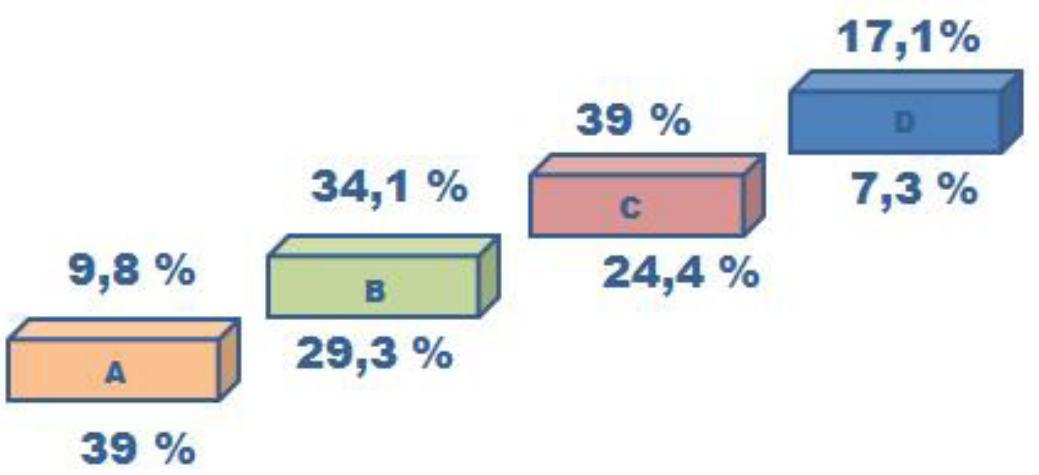

Figura 5. Escalera de aprendizaje para la pregunta 3

Jornadas de Formación e Innovación Docente del Profesorado | № 2 (2019) Esta obra se distribuye con la licencia Creative Commons Reconocimiento-NoComercial-SinObraDerivada 
La cuarta pregunta tenía que servir para dejar claro cuántas horas se pueden usar las lentes de contacto, qué ambientes podían ser hostiles, y con cuáles se puede dormir. El hecho de situarlo o no en un avión no era relevante, excepto por el aire acondicionado, pero es lo que más confusión ha creado, y lo que ha hecho que la mayoría de lo alumnos se sitúe en el tercer nivel y no en el más elevado (figura 7). Además, es la única pregunta en la que después del ciclo aumenta el porcentaje de estudiantes en el escalón de conocimiento más bajo.

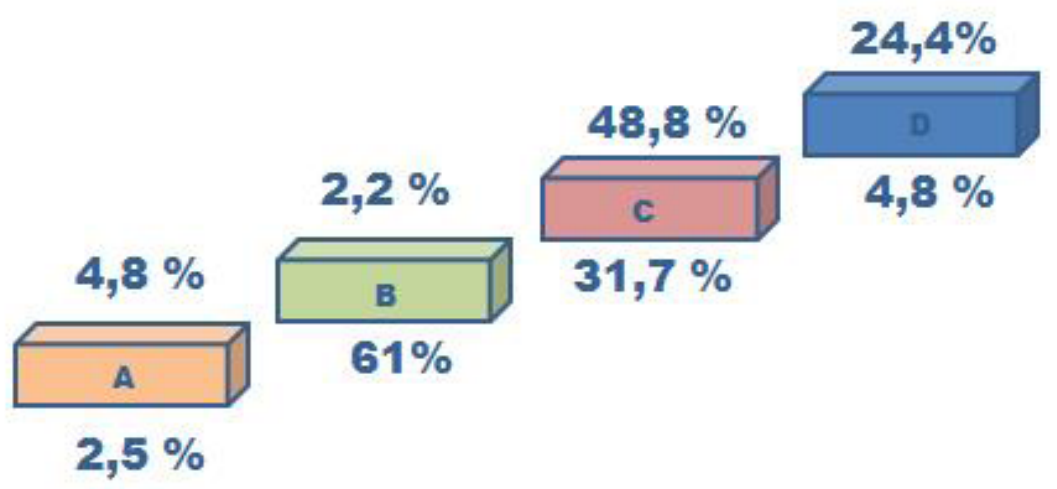

Figura 6. Escalera de aprendizaje para la pregunta 4

Por último, para cerrar el tema de posibles usuarios, se pregunta por los niños. Es la pregunta en la que más evolución ha habido, aunque como comienzo, la mitad de los encuestados tenía claro que sí podían usarlas, los límites impuestos no eran los correctos. El logro está en que en el cuestionario final, practicamente toda la clase se divide a partes iguales entre los dos niveles de conocimientos más altos, superando las barreras de aprendizaje iniciales (figura 8).

Jornadas de Formación e Innovación Docente del Profesorado | № 2 (2019) Esta obra se distribuye con la licencia Creative Commons Reconocimiento-NoComercial-SinObraDerivada 


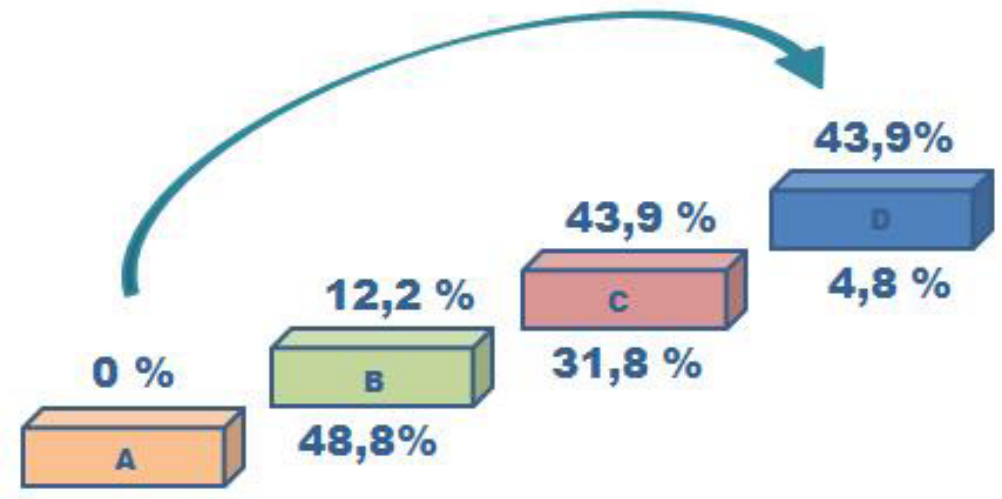

\section{$14,6 \%$}

Figura 7. Escalera de aprendizaje para la pregunta 5

Tras comparar las respuestas del cuestionario inicial con las del final se aprecia que en la mayoría de los alumnos existe una evolución en sus respuestas, aunque siempre existen excepciones, que no solo no suben su nivel, si no que pasan en algunas de sus respuestas de lo más alto a lo más bajo. En general la evolución no ha sido la esperada, esto me hace plantearme si las actividades realizadas han sido las apropiadas pero, sobre todo, dudo sobre las propias preguntas, por lo complicado que ha sido identificar los diferentes niveles mentales y los saltos de conocimientos al analizar las respuestas.

Además, creo que el número de participantes en cada grupo hace que no trabajen todos. Los que evolucionan menos en su aprendizaje pueden ser aquellos que se "esconden" detrás de sus compañeros, por lo que sería algo a mejorar en el ciclo. Tendría que conseguir que en los trabajos en grupo participen todos los integrantes al mismo nivel. 


\section{Evaluación del CIMA}

Los resultados obtenidos en el análisis del cuestionario y reflejados en las escaleras de aprendizaje demuestran que el diseño ha cumplido, en parte, con su cometido, ya que ha mejorado los resultados con respecto al cuestionario inicial, aunque haya sido de forma muy leve en algunos niveles.

Creo que el punto de partida en este CIMA debería tener un nivel más bajo, los alumnos saben teoría sobre lentes de contacto, pero no saben manejarla, por lo que habría que reformular las actividades, para que no solo seleccionen un tipo de lente para un tipo de paciente. Mi planteamiento pasaría por ofrecer todas las posibilidades y los argumentos, lo que les obligaría a aclarar todos los conceptos y no solo a repasar el que al final eligen.

Para mí era algo fundamental que los alumnos participaran en clase, primero por la propia asignatura, si aprendemos a atender a los pacientes y a dar solución a sus demandas, no lo podemos hacer sin analizar casos reales y debatirlos; y segundo, porque pasarme hora y media hablando y viendo cómo los que me deberían escuchar están en otro sitio, solo me deja una sensación de pérdida de tiempo.

Creo que los alumnos no son conscientes de qué tipo de aprendizaje les favorece más, la mayoría sólo quiere saber cómo aprobar, pocos piensan que esos conocimientos los van a tener que poner en práctica en la vida real.

Es importante en mi asignatura trasladar lo impartido en las aulas a casos prácticos reales. Esto me ha hecho reafirmar mi postura como docente, postura que posiciona a los alumnos como lo más importante, no al profesor, y que la interacción entre ambas partes debe ser mayor (Bain, 2007). 
El mapa de contenidos, junto con el diseño de la secuencia de actividades, de forma que se pueda manejar mejor el hilo conductor de una clase, manteniendo la atención y desarrollando trabajos dentro y fuera de la misma y que implique más participación e independencia en el aprendizaje, se han convertido para mí en lo primero a incorporar de forma definitiva en mi método de trabajo, aunque ajustar esta metodología con el tiempo asignado para impartir la asignatura se convierta en el primer inconveniente.

Las preguntas clave, así como los escalones de aprendizaje, siguen suponiendo un reto para mí, que espero poder superar con la aplicación continuada de este método de trabajo.

Por lo tanto, sí que me propongo seguir avanzando en la mejora del modelo metodológico con la introducción en mi rutina de trabajos en grupo y exposiciones, así como con talleres que les hagan llevar la teoría a la realidad de lo que será su práctica diaria.

Resumiendo, la aplicación del CIMA, como parte del Curso General de Docencia Universitaria (CGDU), dentro del Programa de Formación e Innovación Docente del Profesorado 2019 (FIDOP), ha abierto una puerta para afianzar ideas, para desarrollar una nueva metodología de enseñanza basada en los alumnos, que da la vuelta a mi manera de trabajar, ahora sabiendo lo que hago y por qué, para colocar a los estudiantes y el aprendizaje de los mismos en el centro del proceso de enseñanza.

Jornadas de Formación e Innovación Docente del Profesorado | № 2 (2019) Esta obra se distribuye con la licencia Creative Commons 


\section{Referencias bibliográficas}

Bain K. (2007). Lo que hacer los mejores profesores universitarios. Valencia: Publicacions de la Universitat de Valéncia.

Coll, C. et al. (1993). El constructivismo en el aula. Barcelona: Graó.

Cubero, R. (2005). Perspectivas constructivistas. La intersección entre el significado, la interacción y el discurso. Barcelona: Graó.

Finkel D. (2008). Dar clase con la boca cerrada. Valencia: Publicacions de la Universitat de Valéncia.

García Díaz E., Porlán R. y Navarro E. (2017). Los fines y los contenidos de la enseñanza. En R. Porlán (Coord.), Enseñanza Universitaria. Cómo mejorarla, pp. 55-72. Madrid: Morata.

Porlán R. (Coord.) (2017). Enseñanza Universitaria. Cómo mejorarla. Madrid: Morata.

Rivero, A. y Porlán, R. (2017). La evaluación en la enseñanza universitaria. En R. Porlán (Coord.), Enseñanza universitaria. Cómo mejorarla, pp. 73-91. Madrid: Morata. 